\title{
Effects of Aqueous Extract of Purslane (Portulaca oleracea) on Hepatic Enzymes in Two Models of Renal Ischemia-Reperfusion Injury in Rats
}

\author{
Majid Askaripour, ${ }^{1}$ S. Reza Fatemi-Tabatabaei, ${ }^{1}$ Foruzan Hosseini, ${ }^{2, *}$ Masoome Rashno, ${ }^{1}$ and Shahab \\ Ghaderi $^{1}$ \\ ${ }^{1}$ Department of Physiology, Faculty of Veterinary Medicine, Shahid Chamran University, Ahvaz, IR Iran \\ ${ }^{2}$ Department of Physiology, Faculty of Medicine, Kurdistan University of Medical Sciences, Sanandaj, IR Iran \\ "Corresponding author: Foruzan Hosseini, Department of Physiology, Faculty of Medicine, Kurdistan University of Medical Sciences, Sanandaj, IR Iran. E-mail: \\ hosseinif@yahoo.com
}

Received 2014 October 31; Accepted 2015 January 8.

\begin{abstract}
Objectives: This study investigated the effect of purslane on hepatic enzymes following renal ischemia-reperfusion (IR) injury in rats by two methods.

Materials and Methods: In this experimental study, 36 male Wistar rats randomly divided into 6 groups $(\mathrm{n}=6)$ : I) previously nephrectomized control, II) simultaneously nephrectomized control, III) previously nephrectomized + IR, IV) simultaneously nephrectomized + IR, V) previously nephrectomized treated by aqueous extract of P. oleracea L. + IR, VI) simultaneously nephrectomized treated by aqueous extract of P. oleracea L. + IR control and ischemia-reperfusion groups received vehicle and treatment groups received aqueous extract of purslane (300 mg/kg). In model 1, the right kidney was nephrectomized, 20 days prior to experiment, in model 2; nephrectomy was done just before induction of 45 minutes ischemia and 24 hours reperfusion to the left kidney.

Results: Hepatic enzymes were elevated after induction of ischemia reperfusion. Administration of purslane decreased hepatic enzymes.

Conclusions: Purslane prevented liver damage more effectively in simultaneously nephrectomized model.

Keywords: Hepatic Enzymes, Oxidative Stress, Purslane, Remote Organ Injury
\end{abstract}

\section{Background}

Renal ischemia-reperfusion (IR) injury occurs following many clinical settings [1]. Renal IR also results in remote organ dysfunction to the brain, heart, lung and liver $[1,2]$. This multiple organ failure is responsible for high rate of mortality after IR injury [1].

A key factor contributing to the development of liver injury is oxidative stress and subsequent increase in generation of reactive oxygen species. These events lead to increase in lipid peroxidation and reduction in the most antioxidants of the liver [3]. Hepatic enzymes such as alanine aminotransferase (ALT) and aspartate aminotransferase (AST) increase following renal IR and were accepted as a good indicator of liver injury [4].

Purslane (Portulaca oleracea L.) is a well-known plant for its antioxidant properties [5]. Some studies have shown health benefits of $P$. oleracea L. in clinical conditions such as diabetic nephropathy, nephrotoxicity and hepatotoxicity [6]. Thus in this study we tried to evaluate the potential effect of P. oleracea L. on liver injury following renal IR.

There are different methods for induction of renal IR that may lead to remote organ injury [7]. Outcomes of these models could be varying upon site and duration of ischemia induction and reperfusion time [8].

\section{Objectives}

Therefore we examined the effects of the aqueous extract of P. oleracea L. on hepatic enzymes as indicator of liver injury following induction of renal IR in two models.

\section{Materials and Methods}

\subsection{Animals}

In this experimental study, 36 male Wistar rats, 250 $300 \mathrm{~g}$ (from Shahid Chamran university) were kept in 12 hours light-dark cycles at $24 \pm 2^{\circ} \mathrm{C}$ and had ad libitum access to water and food. All ethical codes of working on experimental animals (ministry of health and medical education) were observed. 


\subsection{Preparation of Extract}

Purslane was collected from campus of Ramin university of Ahvaz and was identified by department of pharmacology, Shahid Chamran university. The aerial parts of purslane were cleaned, dried in shadow and powdered by mechanical grinder. Three hundred grams of powder were mixed to $1500 \mathrm{~mL}$ of distilled water. After 72 hours, mixture was centrifuged (1000 g, 20 minutes) and filtered then allowed to its water to be completely evaporated.

\subsection{Experimental Procedure}

Animals randomly divided into 6 groups $(n=6)$ : I) previously nephrectomized control; II) simultaneously nephrectomized control; III) previously nephrectomized + IR; IV) simultaneously nephrectomized + IR; V) previously nephrectomized treated by aqueous extract of $P$. oleracea $\mathrm{L}$. + IR; VI) simultaneously nephrectomized treated by aqueous extract of P. oleracea L. + IR.

Control groups and IR groups received normal saline; treatment groups received $300 \mathrm{mg} / \mathrm{kg}$ of aqueous extract of $P$. oleracea L. for 5 days before experiment by gavage. In model 1, right kidney nephrectomy was performed among groups I, III and V, 20 days before the experiment, in model 2, (groups: IV, V, VI) animals were unilaterally nephrectomized exactly before induction of IR or sham operation to the left kidney. For induction of IR, animals were anaesthetized by ketamine ( $80 \mathrm{mg} / \mathrm{kg}$ ) and xylazine $(10 \mathrm{mg} / \mathrm{kg})$. A longitudinal incision was made and the left renal pedicle was isolated. After a 30 minutes stabilization period, IR injury was performed by clamping of the left renal pedicle. Forty five minutes later the clamp was removed and abdominal wall was closed with 3 - 0 silk. Then after 24 hours (reperfusion period), animals were anesthetized again and blood samples were obtained from aorta and serum samples were kept in $-20^{\circ} \mathrm{C}$ until measurement of the serum for measurement of hepatic enzymes. ALT, AST, alkaline phosphatase (ALP) and lactate dehydrogenase (LDH) were measured by appropriate commercial kits (Pars Azmoon Co., Iran) photometrically.

\subsection{Statistical Analysis}

Statistical analysis was carried out using SPSS-17 software. Data were expressed as mean \pm SEM. Comparisons were made using one-way analysis of variance (ANOVA) followed by LSD test. $\mathrm{P}<0.05$ was considered statistically significant.

\section{Results}

Renal IR injury increased serum ALT level 24 hours after reperfusion in the simultaneously nephrectomized model. Pretreatment with purslane attenuated this elevation significantly. As indicated by comparison between two models, in all groups the ALT levels were higher in the simultaneously nephrectomized rats (Table 1). Indeed not significant changes in ALT were seen between groups in the previously nephrectomized model. The levels of AST were higher in the simultaneously nephrectomized model although not significantly. The alterations of AST among all groups were also nonsignificant (Table 1).

The levels of ALP significantly increased in the previously nephrectomized rats after induction of renal IR. Administration of purslane in this model reduced the serum ALP in comparison to IR group. But it steel was higher than its respective control value (Table 1 ). The concentration of ALP was higher in the simultaneously nephrectomized control group than its value among similar group in the other model. Another interesting change was decrease in ALP among the treated rats in comparison to the respect IR group.

The mean concentration of serum LDH was elevated following induction of the renal IR in the previously nephrectomized rats (Table 1). Pretreatment with purslane did not prevent this elevation effectively. In the other model (simultaneously nephrectomized), the LDH values were high in the control and IR groups and preadministration of purslane attenuated the LDH increase, significantly. Comparison between two models indicated higher LDH concentrations in the control and IR groups of the simultaneously nephrectomized rats.

\section{Discussion}

As indicated by the results of this study, both models of IR showed some degree of liver injury as a remote organ although the simultaneously nephrectomized model produced more prominent alterations. Previous studies reported that renal IR could disturb liver function and morphology $[9,10]$. Kadkhodaee et al. suggested that a minimum of 45 minutes ischemia period is necessary to produce the liver damage as a remote organ [10]. Wang et al. showed that 1 hour ischemia and 4 or 8 hours reperfusion of the kidney increased the serum ALT and AST which seemed to be due to the hepatic cell membrane damage [9].

In the present study the serum concentrations of ALT, ALP and LDH increased significantly following renal IR, but such elevation was not seen in AST level. It believed that the increase of aminotransferase concentrations is due to their enhanced release from damaged or dead hepatocytes and not to an increased enzyme synthesis [11]. Among hepatic enzymes, ALT is largely liver specific, while AST is also found in skeletal, cardiac muscle, brain and many other organs. LDH is also a general marker of tissue injury [11]. 
Table 1. Comparison Between Groups in Terms of Serum ALT, AST, ALP and LDH ${ }^{\mathrm{a}, \mathrm{b}}$

\begin{tabular}{lcccc}
\hline Groups & ALT & AST & ALP & LDH \\
\hline I & $59.99 \pm 5.62$ & $243.45 \pm 23.67$ & $333.32 \pm 16.83^{* * *}$ & $846.75 \pm 26.92^{*}$ \\
II & $126.38 \pm 9.50^{*, \# \# \#}$ & $287.00 \pm 11.99$ & $516.50 \pm 40.16^{\# \# \#}$ & $1451.00 \pm 178.40^{\# \#}$ \\
III & $69.73 \pm 7.58$ & $278.00 \pm 26.67$ & $493.80 \pm 29.46$ & $1259.23 \pm 149.52$ \\
IV & $170.03 \pm 22.13^{\# \# \#}$ & $340.75 \pm 30.46$ & $550.13 \pm 44.08$ & $419.35 \pm 20.26^{*}$ \\
V & $57.62 \pm 3.86$ & $276.75 \pm 20.68$ & $1054.02 \pm 176.66$ & $418.75 \pm 29.08^{*}$ \\
VI & $99.13 \pm 12.98^{\# \#, \$}$ & $274.33 \pm 22.28$ & $1192.83 \pm 136.55^{*}$ & $202.57^{\#}$ \\
\hline
\end{tabular}

${ }^{\mathrm{a}}$ Values are expressed as mean $\pm \mathrm{SEM}$ and unit is presented as $\mathrm{U} / \mathrm{L} .{ }^{*},{ }^{* *}$ and ${ }^{* * *}$ Indicate significant differences vs. respect IR groups $(\mathrm{P}<0.05, \mathrm{P}<0.01$ and $\mathrm{P}<0.001)$. \#, \#\# and \#\#\# Indicate significant differences between similar groups of two models $(\mathrm{P}<0.05, \mathrm{P}<0.01$ and $\mathrm{P}<0.001)$.

bI, priory nephrectomized control; II, simultaneously nephrectomized control; III, previously nephrectomized + IR; IV, simultaneously nephrectomized + IR; V, previously nephrectomized treated by aqueous extract of P. oleracea L. + IR; VI, simultaneously nephrectomized treated by aqueous extract of $P$. oleracea L. + IR.

Then we concluded that induction of renal IR in this study caused remote organ injury in the liver and probably in the other organs.

The pre-treatment with purslane significantly prevented the ALT, ALP and LDH increase in the simultaneously nephrectomized model. In the other model of experiment, purslane had such effect only on the serum ALP concentration that could be expectable because of the less enzymatic changes seen after 24 hours of reperfusion in that model.

The hepatoprotective activity of purslane was reported in some situations like ligation-induced hepatic fibrosis [12], cisplatin-induced toxicity [13], and carbon tetrachloride-induced hepatotoxicity [14]. Purlane is well known for the potent antioxidant activity [15]. As the oxidative stress and free radical formation have a key role in propagation of remote organ injury, the preventive effect of purslane in the liver injury after renal IR in the present study seems to be logical.

As the results showed, the consequence of the renal IR was apparently different in the two models of experiments. In the previously nephrectomized animals the effects of the induction of renal IR and administration of purslane on the most of the measured hepatic enzymes were not significant. Comparisons between two models showed notable differences. Then it seems that the judgment about benefit of the purslane treatment could affect by the method used for induction of the IR injury. In conclusion, purslane as an antioxidant ameliorated remote organ injury in the liver after renal IR and this effect was seen more clearly in the simultaneously nephrectomized model, which could be in part because of more injury occurred in that model.

\section{Acknowledgments}

The authors would like to thank the financial support provided by the vice-presidency of research of Shahid Chamran university (grant number 9227055).

\section{Footnotes}

Authors' Contribution: S. Reza Fatemi-Tabatabaei designed the study; Majid Askaripour, Masoome Rashno and Shahab Ghaderi performed experiments; Foruzan Hosseini performed data analysis and wrote the paper.

Funding/Support: Shahid Chamran University, Ahvaz, Iran.

\section{References}

1. White LE, Hassoun HT. Inflammatory Mechanisms of Organ Crosstalk during Ischemic Acute Kidney Injury. Int J Nephrol. 2012;2012:505197. doi: 10.4061/2012/505197. [PubMed: 21826270].

2. Grams ME, Rabb $H$. The distant organ effects of acute kidney injury. Kidney Int. 2012;81(10):942-8. doi: 10.1038/ki.2011.241. [PubMed: 21814177].

3. Yap SC, Lee HT. Acute kidney injury and extrarenal organ dysfunction: new concepts and experimental evidence. Anesthesiology. 2012;116(5):1139-48. doi:10.1097/ALN.0b013e31824f951b. [PubMed: 22415388].

4. Golab F, Kadkhodaee M, Zahmatkesh M, Hedayati M, Arab H, Schuster R, et al. Ischemic and non-ischemic acute kidney injury cause hepatic damage. Kidney Int. 2009;75(8):783-92. doi: 10.1038/ki.2008.683. [PubMed: 19177157].

5. Cai Y, Luo Q, Sun M, Corke H. Antioxidant activity and phenolic compounds of 112 traditional Chinese medicinal plants associated with anticancer. Life Sci. 2004;74(17):2157-84. doi: 10.1016/j.lfs.2003.09.047. [PubMed: 14969719].

6. Okafor IA, Ayalokunrin MB, Orachu LA. A review on Portulaca oleracea (Purslane) plant-Its nature and biomedical benefits. Int J Biomed Res. 2014;5(2):75-80. 
7. Gultekin SS, Odabas O, Ginis Z, Gokce A, Yigman M, Dogan S, et al. Scintigraphic comparison of renal ischemia-reperfusion injury models in rats: correlations with biochemical and histopathological findings. Ann Nucl Med. 2013;27(6):564-71. doi: 10.1007/s12149-013-0727-3. [PubMed: 23575512].

8. Kurian GA, Pemaih B. Standardization of in vitro Cell-based Model for Renal Ischemia and Reperfusion Injury. Indian J Pharm Sci. 2014;76(4):348-53. [PubMed: 25284933].

9. Wang B, Bai M, Bai Y, Li Q. Liver injury following renal ischemia reperfusion in rats. Transplant Proc. 2010;42(9):3422-6. doi: 10.1016/j.transproceed.2010.09.008. [PubMed: 21094789].

10. Kadkhodaee M, Golab F, Zahmatkesh M, Ghaznavi R, Hedayati M, Arab HA, et al. Effects of different periods of renal ischemia on liver as a remote organ. World J Gastroenterol. 2009;15(9):1113-8. [PubMed: 19266605].

11. Dancygier H. Basic laboratory parameters. In: Dancygier $\mathrm{H}$, editor.
Clinical hepatology. USA: Springer; 2010. pp. 319-32.

12. Ali SI, Said MM, Hassan EK. Prophylactic and curative effects of purslane on bile duct ligation-induced hepatic fibrosis in albino rats. Ann Hepatol. 2011;10(3):340-6. [PubMed: 21677337].

13. Sudhakar D, Krishna Kishore R, Parthasarathy PR. Portulaca oleracea L. extract ameliorates the cisplatin-induced toxicity in chick embryonic liver. Indian J Biochem Biophys. 2010;47(3):185-9. [PubMed 20653291].

14. Anusha M, Venkateswarlu M, Prabhakaran V, Taj SS, Kumari BP, Ranganayakulu D. Hepatoprotective activity of aqueous extract of Portulaca oleracea in combination with lycopene in rats. Indian JPharmacol. 2011;43(5):563-7. doi: 10.4103/0253-7613.84973. [PubMed: 22022001].

15. Uddin MK, Juraimi AS, Hossain MS, Nahar MA, Ali ME, Rahman MM Purslane weed (Portulaca oleracea): a prospective plant source of nutrition, omega-3 fatty acid, and antioxidant attributes. Sci World J. 2014;2014:951019. doi: 10.1155/2014/951019. [PubMed: 24683365]. 